\title{
Emerging Multidrug-Resistant Hybrid Pathotype Shiga Toxin-Producing Escherichia coli 080 and Related Strains of Clonal Complex 165, Europe
}

\author{
Aurélie Cointe, André Birgy, Patricia Mariani-Kurkdjian, Sandrine Liguori, Céline Courroux, \\ Jorge Blanco, Sabine Delannoy, Patrick Fach, Estelle Loukiadis, Philippe Bidet, Stéphane Bonacorsi
}

Enterohemorrhagic Escherichia coli serogroup O80, involved in hemolytic uremic syndrome associated with extraintestinal infections, has emerged in France. We obtained circularized sequences of the 080 strain RDEx444, responsible for hemolytic uremic syndrome with bacteremia, and noncircularized sequences of $35080 \mathrm{E}$. coli isolated from humans and animals in Europe with or without Shiga toxin genes. RDEx444 harbored a mosaic plasmid, pR444_A, combining extraintestinal virulence determinants and a multidrug resistanceencoding island. All strains belonged to clonal complex 165, which is distantly related to other major enterohemorrhagic $E$. coli lineages. All stx-positive strains contained eae- $\xi$, ehxA, and genes characteristic of pR444_A. Among stx-negative strains, 1 produced extended-spectrum $\beta$-lactamase, 1 harbored the colistin-resistance gene $m c r 1$, and 2 possessed genes characteristic of enteropathogenic and pyelonephritis E. coli. Because O80-clonal complex 165 strains can integrate intestinal and extraintestinal virulence factors in combination with diverse drug-resistance genes, they constitute dangerous and versatile multidrug-resistant pathogens.

$\mathrm{E}$ nterohemorrhagic Escherichia coli (EHEC), a subset of $\checkmark$ Shiga toxin-producing E. coli (STEC), are major foodborne pathogens responsible for outbreaks and sporadic cases of gastrointestinal diseases ranging from simple diarrhea to hemorrhagic colitis, characterized by bloody diarrhea. The

Author affiliations: Hôpital Robert-Debré (AP-HP), Paris, France (A. Cointe, A. Birgy, P. Mariani-Kurkdjian, S. Liguori, C. Courroux, P. Bidet, S. Bonacorsi); Université Paris Diderot, Sorbonne Paris Cité, Paris (A. Cointe, A. Birgy, P. Mariani-Kurkdjian, P. Bidet, S. Bonacorsi); Universidade de Santiago de Compostela, Lugo, Spain (J. Blanco); ANSES, Plateforme IdentyPath, Maisons-Alfort, France (S. Delannoy, P. Fach); Université de Lyon, CNRS, INRA, UCBL, VetAgro Sup, Laboratoire d'Écologie Microbienne, Villeurbanne, France (E. Loukiadis)

DOI: https://doi.org/10.3201/eid2412.180272 most serious complication, particularly in young children, is hemolytic uremic syndrome (HUS), defined by a combination of renal failure, thrombocytopenia, and hemolytic anemia (1). Post-STEC HUS is a major worldwide public health concern because it is the primary cause of acute renal failure in children (1). These clinical features result mainly from the action of the phage-encoded Shiga toxin (Stx), of which there are 2 types: Stx1, which has 3 subtypes, Stx1a, 1c, and 1d; and Stx2, which has 7 subtypes, Stx2a-g. In typical EHEC, adhesion to the intestinal epithelium is mediated by the locus of enterocyte effacement (LEE), a chromosomal pathogenicity island (PAI), shared with Enteropathogenic E. coli (EPEC) strains, which encodes a type III secretion system (T3SS), an adhesin called intimin, and its receptor Tir. Intimin, encoded by the eae gene, is a major virulence factor (VF) involved in the intimate attachment of typical EHEC to intestinal epithelium, causing characteristic attaching and effacing lesions. EHEC enterohemolysin $(e h x A)$ is a pore-forming cytolysin carried by a plasmid involved in EHEC virulence. This plasmid, initially described as part of the O157 serogroup (pO157) (2), can carry 2 additional VFs, a catalase peroxidase, encoded by katP, and a serine protease, encoded by esp $P$, which can cleave human coagulation factor $\mathrm{V}$ and might be involved in the development of hemorrhagic colitis (3).

Serogroup O157 is the predominant STEC serogroup worldwide, but non-O157 serogroups are increasingly associated with post-STEC HUS, and the unusual serogroup O80 is emerging in France and Europe. In 2016, O80 represented the second most frequent serogroup isolated in France, after serogroup O26 (4). This phenomenon is no longer restricted to France; strains of serotype O80:H2, all belonging to sequence type (ST) 301, have been identified in Spain (5), the Netherlands (6), and Switzerland (7).

This serogroup is unique for several reasons. First, it is always associated with multiple determinants of resistance 
(i.e., resistance to aminopenicillin, aminoglycoside, nalidixic acid, cotrimoxazole, tetracycline, or phenicols), whereas a resistance phenotype is uncommon among EHECs, which are generally fully susceptible to antibiotics, except for rare clones, such as the epidemic O104:H4 German clone carrying a $b l a_{\mathrm{CTX}-\mathrm{M}-15}$ gene (8). Furthermore, unusual extraintestinal infections have recently been described for this serogroup $(9,10)$, such as bacteremia, whereas EHEC is generally known to be a strictly intestinal pathogen. A recent case in the Netherlands illustrates the potential extreme pathogenicity of this serogroup; a 16-month-old boy died from multiorgan failure and extensive cerebral thrombotic microangiopathy attributable to an O80 Stx2d-producing E. coli strain (6). Finally, O80 EHEC appears to be a hybrid pathotype that combines intestinal VFs (Shiga toxin $[s t x]$, intimin [eae], enterohemolysin $[e h x A]$ ) and extraintestinal VFs (aerobactin [iucC]; salmochelin [iroN], an iron uptake protein encoded by sitABCD; serum resistance protein $[i s s p]$; a putative secretion system I [ets C]; omptin [ompTp]; hemolysin [hlyF], and 2 bacteriocins $[\mathrm{cia}$ and $\mathrm{cva}]$ ), suggesting the presence of a pS88-like plasmid (11). pS88 is a ColV plasmid, a key determinant of extraintestinal pathogenic E. coli virulence in poultry and humans. This plasmid is involved in neonatal meningitis (11) and could explain the occurrence of extraintestinal dissemination in these EHEC infections. The recent diffusion in Europe, high potential extraintestinal pathogenicity, and multidrug resistance (MDR) of this hybrid pathotype led us to further characterize these strains, which might represent a major public health concern.

\section{Methods}

We further characterized O80:H2 EHEC by fully sequencing a recent representative strain, called RDEx444. RDEx444 was responsible for a highly severe case of post-STEC HUS, complicated by bacteremia, in an 8-month-old infant in February 2016 in Bourg-en-Bresse, France. Initial symptoms were febrile diarrhea with signs of sepsis. Blood and stool cultures were positive for O80 EHEC, but urine cultures remained negative, suggesting gut translocation that led to bacteremia. The appearance of biologic signs of HUS with oliguria necessitated transfer to intensive care, several blood transfusions, and 5 days of peritoneal dialysis.

We performed sequencing of RDEx444 by using the PacBio single-molecule real-time method with RS II chemistry 2.4.0 (Pacific Biosciences, Menlo Park, CA, USA); we used 1 single-molecule real-time cell. De novo assembly was performed twice by using the HGAP pipeline (https://github.com/PacificBiosciences/BioinformaticsTraining/wiki/HGAP) and annotation by using the MAGE platform (http://www.genoscope.cns.fr/agc/microscope/ home/index.php) (Figure 1). For this strain, we performed plasmid typing by using databases (PlasmidFinder 1.3 and

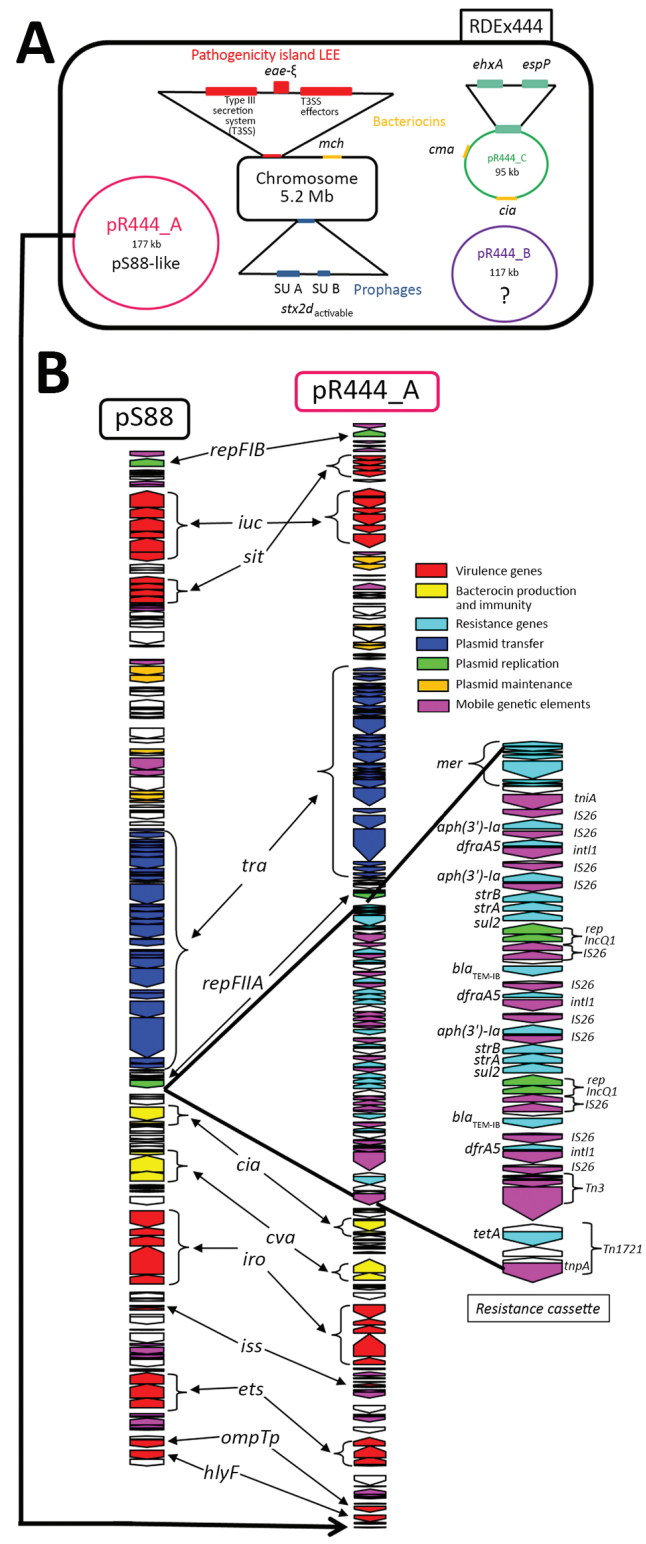

Figure 1. Genetic characterization of RDEx444, a strain of Escherichia coli serotype $080: \mathrm{H} 2$ isolated in France in February 2016 and involved in hemolytic uremic syndrome with bacteremia, carrying both intestinal and extraintestinal virulence factors associated with (multidrug-resistance determinants $(A)$ and genetic comparison between plasmid pS88 and mosaic plasmid pR444_A (B). A) Four circularized contigs (chromosome of 5,256,050 bp and the 3 plasmids pR444_A [176,500 bp], pR444_B [117,090 bp], and pR444_C [95,050 bp]) obtained by using PacBio (Pacific Biosciences, Meno Park, CA, USA) sequencing of RDEx444 are schematically represented. The main virulence factors are presented as colored rectangles. Intestinal virulence factors are indicated in red for the locus of enterocyte effacement genes, blue for prophage-encoded Shiga toxin genes, and green for VFs carried by pR444_C, a pO157-like plasmid. Bacteriocin genes ( $m c h$, cia, and $\mathrm{cma}$ ) are indicated in yellow. B) Comparison of the sequences of pR444_A and pS88, the plasmid of strain S88 involved in neonatal meningitis. LEE, locus of enterocyte effacement. 
pMLST 1.4 [12]) available on the Center for Genomic Epidemiology (CGE) website (http://www.genomicepidemiology.org). Plasmids were also characterized by S1 nuclease pulsed-field gel electrophoresis (PFGE) with Southern hybridization and conjugability of the largest confirmed by experiments using rifampin-resistant $E$. coli $\mathbf{J} 53$, as previously described ( 9 ).

We also sequenced a representative set of 35 O80 strains from various sources and countries using the Nextera kit (Illumina, San Diego, California, USA) to prepare the library. Sequencing was performed by using a MiSeq reagent kit V3 600 cycles (Illumina). This panel included human isolates from France $(\mathrm{n}=21)$, Spain $(\mathrm{n}=3)$, and Switzerland $(\mathrm{n}=2)$, as well as animal and environmental strains isolated in France $(n=3)$, Spain $(n=1)$, Slovakia ( $=1)$, and Germany $(n=4)$ (Figure 2). Some of the human strains from France and strains from Spain and Slovakia have been partially characterized previously (10). The strains from Spain and Slovakia were isolated during 1998-2012 and the strains from France during 2010-2016. The dates of isolation of the strains from Switzerland and Germany were not available. We performed assembly by using CLC Genomics Workbench software and SPAdes, also available on CGE website. The RAST server (http:// rast.nmpdr.org) was used for genome annotation and the PHASTER web server (http://phaster.ca) to identify and annotate prophage sequences within bacterial genomes and plasmids. We established phylogeny by single-nucleotide polymorphism alignments between the contigs generated by CLC Genomics of O80 strains and 9 reference EHEC strain sequences of major serotypes available in GenBank (O157:H7 EDL933, O26:H11 11368, O111:H- 11128,

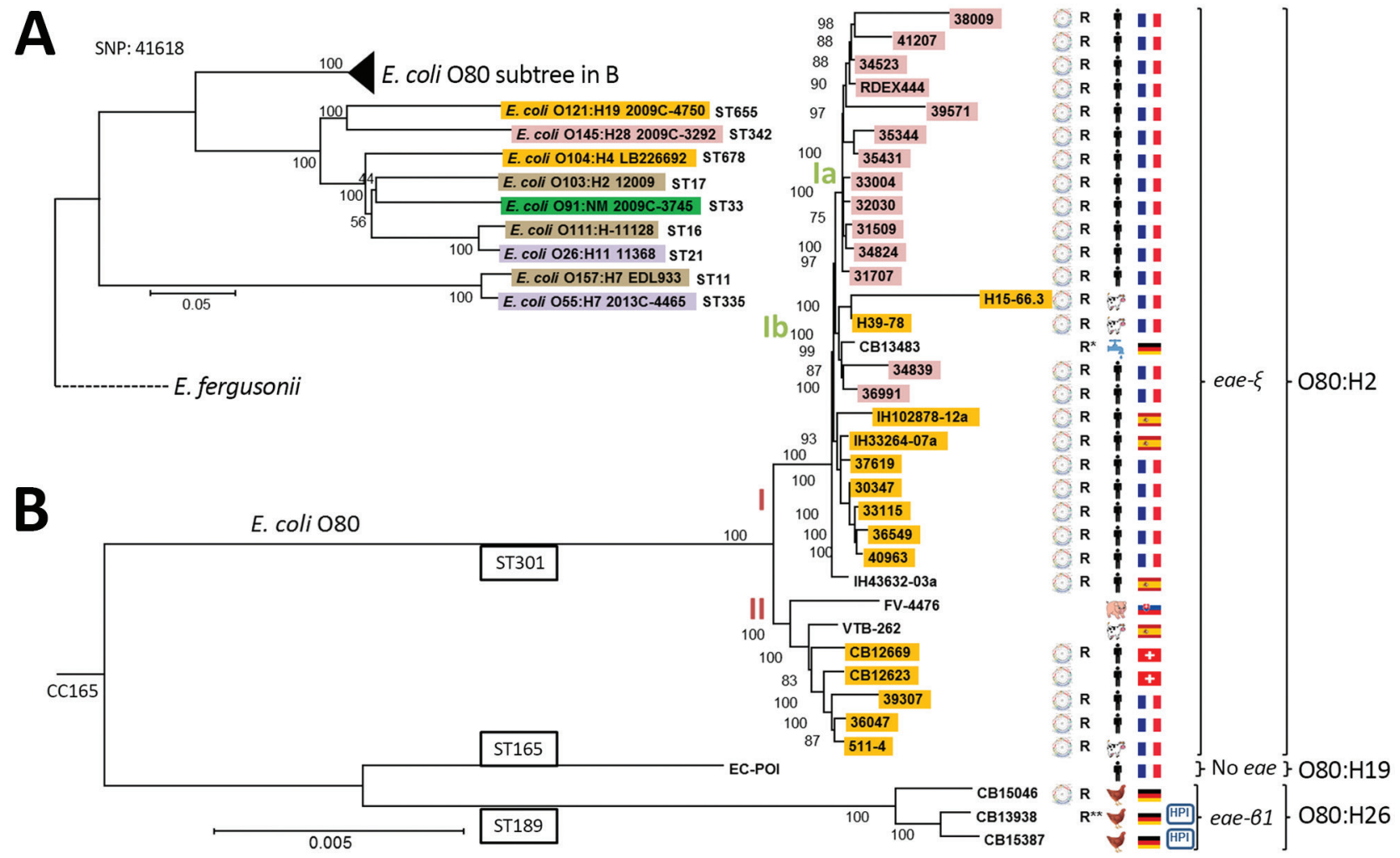

Figure 2. Phylogeny of 36 Escherichia coli serogroup 080 strains isolated from various sources and countries in Europe during 19982016 and their relationship to major enterohemorrhagic $E$. coli lineages. General phylogenic tree rooted on $E$. fergusonii (GenBank accession no. NC_011740), showing (A) the position of O80 strains among major enterohemorrhagic E. coli lineages (O157:H7 EDL933 NC_002655.2, O26:H11 11368 NC_013361.1, O111:H- 11128 NC_013364.1, O103:H2 12009 NC_013353.1, O55:H7 2013C-4465 CP015241, O91:NM 2009C-3745 JHGW00000000, O104:H4 LB226692 EO104H4LB.1, O145:H28 2009C-3292 JHHD00000000, and O121:H19 2009C-4750 JHGL00000000) and (B) a focused view of clonal complex 165, including O80 strains. The highlighted strains carry the Shiga toxin genes; the subtype of Stx is indicated by a color code as follows: purple, stx1a; yellow, stx2a; pink, stx2d ${ }_{a c t i v a b l e}$; brown, stx1a2a; dark green, stx1a2b. The presence of the pS88 like-plasmid is represented by using a plasmid scheme next to the strain number. $R$ to the right of the plasmid indicates that the strain possesses $\geq 2$ resistance genes that confer resistance to $\beta$-lactams, kanamycin, or cotrimoxazole $\left(R^{*}\right.$ indicates presence of additional extended-spectrum $\beta$-lactamase gene; $R^{* *}$ indicates presence of $m c r-1$ gene conferring additional resistance to colistin). The strain origin (country and source of isolation) is represented by flags and human, animal, or water symbols. CC, clonal complex; HPI, high-pathogenicity island (presence of chromosomal locus encoding the siderophore yersiniabactin); SNP, single-nucleotide polymorphism; ST, sequence type. Scale bar indicates nucleotide substitutions per site. 
O103:H2 12009, O55:H7 2013C-4465, O91:NM 2009C3745, O104:H4 LB226692, O145:H28 2009C-3292, and O121:H19 2009C-4750 [online Technical Appendix, https://wwwnc.cdc.gov/EID/article/24/12/18-0272Techapp1.pdf]) using CSIPhylogeny 1.4 (13). The neighbor-joining tree was built by using MEGA 3.1 (https:// www.megasoftware.net) using bootstraps calculated from 100 replicates. The maximum-likelihood method yielded the same results (online Technical Appendix Figure 1).

We performed multilocus sequence typing and identification of acquired antimicrobial resistance genes for all strains by using suitable databases available on the CGE website (SerotypeFinder 1.1 [14], MLST 1.8 [15], and ResFinder 3.0 [16]). Investigation of the resistome was completed by using the Resistance Gene Identifier at the Comprehensive Antibiotic Resistance Database website (https://card.mcmaster.ca/analyze/rgi). Moreover, 166 genes of intestinal and extraintestinal VFs (list available from authors) were assessed by local BLAST+ 2.2.31 analysis (https://blast.ncbi.nlm.nih.gov). Complete results are described in online Technical Appendix Figure 2. Nucleotide sequences of the 36 sequenced O80 strains (complete nucleotide sequence of RDEx444 and 35 draft nucleotide sequences) have been deposited in Genbank (project no. PRJNA449634); accession numbers are available in the online Technical Appendix.

\section{Results}

RDEx444 can be considered to be representative of hybrid pathotype strains because it was responsible for an extraintestinal infection and showed similar virulence and resistance profiles as other strains described elsewhere $(9,10)$. Complete sequencing yielded $2.3 \times 10^{9} \mathrm{bp}$ with 165,041 reads and an N50 (the length of the smallest contig among the set of the largest contigs that together cover $>50 \%$ of the assembly) read length of 19,839 bp.

Four circularized contigs were obtained, including the chromosome of 5,256,050 bp, containing 5,146 coding sequences (CDSs), with an overall $\mathrm{G}+\mathrm{C}$ content of $50.7 \%$ and 3 plasmids of 176,500 (pR444_A), 117,090 (pR444_B), and 95,050 bp (pR444_C). The number and size of the RDEx444 plasmids were corroborated by S1 nuclease PFGE (data not shown). We compiled a schematic representation of the genetic content, including major VFs, of this strain (Figure 1, panel A).

As expected, RDEx444 belongs to serotype O80:H2 and ST301. Among the 12 prophage regions identified on its chromosome, this strain carries a complete Stx-converting bacteriophage of $43.9 \mathrm{~kb}$. RDEx444 carries the $s t x 2 d_{a c-}$ tivable variant, which has been shown to be predictive of severe clinical outcomes and progression to HUS (17). The Stx prophage is integrated into the chromosomal yecE gene, a known integration site $(18,19)$, initially reported in a strain producing Stx2e, encoded by the phage $\varphi \mathrm{P} 27$ (20). The $\approx 15$ $\mathrm{kb}$ region between Stx subunit B and the phage integrase, comprising proteins for DNA replication and repression, shares strong homology with the phage $\varphi \mathrm{P} 27$. RDEx444 is otherwise a typical EHEC, given that it harbors a complete LEE and does not possess atypical adhesins, such as autoagglutinating adhesion (saa) or aggregative factors ( $a g g A / R)$. RDEx 444 carries the rare variant of the intimin gene $e a e-\xi$ (5), which has only very rarely been observed among other EHEC serotypes, but which is shared by all previously described O80 EHEC and EPEC isolates $(5-7,9,10)$. Two other chromosomal traits of RDEx444 might also confer a selective advantage: 1) a region encoding microcin $\mathrm{H} 47$ ( $m c h A, S, X, B, C, D, E$, and $F$ ) $\approx 35 \mathrm{~kb}$ downstream from the Stx prophage, and 2) a chromosomal mutation in DNA gyrase (gyrA $S 83 L$ ), which confers quinolone resistance.

Plasmid pR444_C is 95,050 bp long with a $\mathrm{G}+\mathrm{C}$ content of $49.8 \%$ and was predicted to contain 114 CDSs. Plasmid pR444_C is comparable to pO157 because it contains enterohemolysin $(e h x A)$ and serine protease $(e s p P)$ but does not possess catalase peroxidase (katP). Moreover, 2 other colicines, Ia (cia) and M ( $\mathrm{cma}$ ), are also present on this plasmid.

Plasmid pR444_B is 117,090 bp long with a G+C content of $46.5 \%$ and contains $135 \mathrm{CDSs}$ and, unexpectedly, those for 3 tRNAs (threonine, asparagine, and tyrosine) redundant with those still present on the chromosome. This plasmid can be considered to be cryptic because it carries no drug resistance or virulence-associated genes, but several phage proteins are present. pR444_B shares high homology (99\% nucleotide identity and $89 \%$ coverage) with plasmid pECOH89 (21), encoding an extended-spectrum $\beta$-lactamase $b l a_{\text {CTX-M-15 }}$ identified in an E. coli strain and belonging to the family of phage-like plasmids. Members of this family are generally untypeable, nonconjugative, and cryptic plasmids, because no known virulence or resistance genes have been identified. Their function is unknown, but they all have strong homology to the Salmonella-specific bacteriophage SSU5 (21).

Plasmid pR444_A is 176,500 bp long, with a $\mathrm{G}+\mathrm{C}$ content of $51.8 \%$, and carries 2 replicons: FII_A and FIB_1 (ST [F2:A-:B1]). We identified 202 CDSs. This plasmid carries virulence-associated genes characteristic of $\mathrm{pS} 88$ (described previously) and MDR genes, and thus can be considered to be a mosaic plasmid. We identified an MDRencoding region $(48,237 \mathrm{bp})$ in addition to the plasmid-related function (56,106 bp) and virulence-associated domain ( $72,157 \mathrm{bp})$, closely related to pS88 (99\% nucleotide identity and $96 \%$ query coverage) (11). This MDR-encoding region contains genes encoding resistance tetracycline (tet $A)$, trimethoprim ( $d f r A 5)$, sulfonamide (sul2), $\beta$-lactam (blaTEM-IB $)$, kanamycin ( $\left.a p h\left[3^{\prime}\right]-I a\right)$, and streptomycin (strA and $s t r B$ ) and resistance against heavy metals such as mercury 
(mer gene) (Figure 1, panel B). This resistance cassette has only been described for pO26-CRL ${ }_{125}$ (100\% nucleotide identity, 98\% coverage) from an O26 EHEC but without VFs (22). Co-localization of extraintestinal VFs and MDR genes on the same plasmid was confirmed by Southern hybridization (data not shown). Moreover, we confirmed that this plasmid is conjugative, suggested by the presence on the annotation of an almost complete F-like transfer region, by successfully conjugating it with rifampin-resistant $E$. coli J53 (data not shown). Thus, this plasmid can diffuse by horizontal transfer.

Accordingly, this mosaic plasmid shows high homology with plasmid pS88, responsible for extraintestinal virulence in neonatal meningitis strains within which a resistance cassette has been integrated (Figure 1, panel B). Furthermore, pR444_A also carries 2 bacteriocin genes (cia and $c v a$ ), such as pS88, which might confer a selective advantage by promoting intestinal establishment and colonization by killing other E. coli and freeing up their ecologic niches (Figure 1, panel B).

We sequenced a panel of strains consisting of representative O80 strains from France and all O80 strains from outside of France available at the beginning of the study to establish the genetic relationship between O80 strains isolated in several countries in Europe and analyze the diversity of their genetic content. We obtained an average of 233 contigs, with a mean depth of coverage of $58 \mathrm{X}$ and a mean N50 of 72,312. Statistics of each sequenced genome are summarized in online Technical Appendix Table 1. Singlenucleotide polymorphism analysis $(41,618$ sites total) between the 36 sequenced O80 strains and 9 EHEC strains of other major serotypes (O157:H7, O26:H11, O111:H-, O103:H2， O55:H7， O91:NM， O104:H4， O145:H28, O121:H19) enabled us to establish a phylogenic tree (Figure 2), which shows 2 main clusters. EHEC O157:H7 (EDL933) and O55:H7 (2013C-4465) group together, as expected by their common origin, demonstrated by Feng et al. (23), and are distantly related to the other major EHEC serotypes, including the O80 strains. However, O80 isolates clearly form a separate group, suggesting that O80 emerged independently from the other serogroups. All of the O80 strains belong to clonal complex 165 (CC165), containing ST301, ST165, and ST189 (Figure 2, panel B).

Almost all of the O80 strains (32/36), including RDEx444, belong to the ST301 clonal group of serotype O80:H2. All of these isolates have EHEC markers, including genes encoded by the LEE, containing the rare variant of intimin eae- $\xi$, ehxA, and $s t x 2 a$ or $s t x 2 d$ genes, except for 4 strains missing the stx gene (CB13483, IH43632-03a, FV-4476, and VTB262) (online Technical Appendix Figure 2). However, the presence of the other EHEC markers in these 4 strains (eae, ehxA/espP, or katP) suggests that they were initially STEC and underwent subsequent elimination of the stx gene. This finding led us to search for a scar of the Stx-converting bacteriophage at the insertion site described in the RDEx444 strain (yecE). We first searched for the contig carrying the yecE gene. Then, we used the Phaster webserver system to detect phage regions, which were finally blasted against RDEx 444 . We found scars of $\approx 31 \mathrm{~kb}$ in the human strain from Spain (IH43632$03 \mathrm{a})$ and scars of $\approx 17 \mathrm{~kb}$ in the porcine strain from Slovakia (FV-4476), similar to the RDEx444 Stx-converting bacteriophage (92\% and 94\% nucleotide identity and $40 \%$ and $56 \%$ coverage, respectively) (data not shown). We found no scars for the other 2 strains, suggesting either complete prophage excision or insertion at another site.

All strains carrying the stx gene (28/36) also possess VFs typical of pS88, and all but 1 (CB12623) also carry genes conferring resistance to $\geq 1$ of penicillin, tetracycline, kanamycin, or cotrimoxazole. The consistent association between extraintestinal VFs typical of pS88 and MDR genes, irrespective of the source or country of isolation, might suggest the presence of a mosaic plasmid, such as in the RDEx444 strain. Although it lacks pS88 markers, the water strain from Germany (CB13483) is nevertheless multidrug resistant and carries an extended spectrum $\beta$-lactamase gene $\left(b l a_{\text {СТХ-М-1 }}\right)$, as well as the $m p h A$ gene, which confers resistance to azithromycin, the only antimicrobial drug that can be used for intestinal decontamination of EHEC (24; online Technical Appendix Figure 2).

Among our panel, 3 STEC strains from cattle in France clearly belong to this ST301 clonal group. Cattle might thus represent an animal reservoir for these hybrid pathotype strains, given that the isolates possess exactly the same VFs (eae, genes of T3SS, ehxA, stx, and determinants of the pS88-like plasmid) and resistance genes as human EHECs.

Two clusters (I and II) can be distinguished within the ST301 group we describe. The main difference between these 2 groups concerns the presence of the unknown cryptic plasmid (pR444_B) in RDEx444. All strains of cluster I $(n=25)$ possess $\geq 85 \%$ of the genetic determinants of this plasmid of unknown function. BLAST results are depicted for each strain in online Technical Appendix Table 2. Conversely, no strain of group II $(n=7)$, except 1 , has this plasmid. No strains of ST165 or ST189 carry it either.

Within cluster I, the pS88-like plasmid carries 2 distinct gene profiles, showing its plasticity. The $12 \mathrm{Stx} 2 \mathrm{~d}_{\text {acti- }}$ vable EHECs isolated from humans in France (designated as subcluster Ia in Figure 2) carry the most complete form of the plasmid, identical to pS88. The aerobactin iron-uptake system, encoded by iuc genes, and the type I secretion system, encoded by ets $C$, are absent from the pS88-like plasmid of the subcluster Ib strains.

The second clonal group, ST165, is formed by 1 strain (EC_POI) of serotype O80:H19, which is devoid of all VF and resistance genes. This isolate might reflect the ancestral 
origin from which serogroup O80 EHEC strains were derived after the acquisition of diverse VFs.

Finally, clonal group ST189 consists of the 3 avian strains of serotype O80:H26 from Germany. None has stx or ehxA genes, but all have a complete LEE with the variant $\beta 1$ of the intimin gene (eae). However, none of these strains carries the plasmid $b f p$ gene of typical EPEC (online Technical Appendix Figure 2). One strain (CB13938) has a region of $\approx 27 \mathrm{~kb}$ at the same insertion site (yecE gene) that shares homology with RDEx444 Stx-converting bacteriophage ( $88 \%$ nucleotide identity, $16 \%$ coverage), suggesting a potential scar of Stx-converting phage. Thus, this strain could have been originally an EHEC which secondarily lost its stx gene. No similar regions were found in the other 2 strains of this group. Otherwise, 1 strain (CB15046) has several VFs, confirming the presence of the pS88-like plasmid with MDR genes. The other 2 strains (CB15387 and CB13938) carry the locus encoding the siderophore yersiniabactin $(f y u A)$, also called the high-pathogenicity island; these 2 strains also have pyelonephritis-associated pili with PapGII adhesin (online Technical Appendix Figure 2). These strains constitute another type of hybrid pathotype with intestinal and extraintestinal VFs. Although none of them has VFs typical of pS88 plasmids, CB13938 is multidrugresistant and carries the recently described $\mathrm{mcr}-1$ plasmid gene (25), conferring resistance to colistin. Blast analysis performed with the contig containing $m c r-1$ (10.119 bp) shows strong homology (100\% coverage, $99 \%$ identity) with 2 chromosomal insertion sites previously described in strains Mbl323 and Mbl506 (26).

\section{Discussion}

We deciphered the molecular characteristics of O80:H2CC165 EHEC, an emerging hybrid pathotype diffusing throughout Europe. This pathotype is armed to spread by means of a conjugative plasmid combining extraintestinal virulence with resistance to nearly all major classes of antibiotics, improved by the presence of several plasmid and chromosomally encoded bacteriocins, such as colicines I, V, M, and H47. We used the same criteria of MDR as a recent study in England (27) (bla $a_{\mathrm{TEM}-1}$, str $A$ strB, sull/sul2/dfrA, and tetA) and showed that $93 \%$ $(26 / 28)$ of O80 STEC have this genotypic resistance profile, whereas only $5 \%$ of the strains identified within the O157 and O26 serogroups in the study in England had such a profile. MDR observed with this hybrid pathotype might complicate patient care, and the use of antimicrobial drugs during EHEC infections is still a subject of debate (28). However, the occurrence of invasive infections, such as bacteremia during EHEC infections, with this clone warrants antimicrobial treatment for such infections. In a previous study, the observed Stx rate was lower with a combination of azithromycin and ceftriaxone assays relative to basal secretion, and we proposed this association for the treatment of such infections (10).

Such a troubling plasmid has never been identified in human EHEC isolates. The only example of a similar mosaic plasmid was reported for S. enterica serovar Kentucky, in which an AmpC $\beta$-lactamase gene ( $\left.b l a_{\mathrm{CMY}-2}\right)$ was integrated into a pS88-like plasmid (29). The insertion of an MDR-encoding island in a pS88-like plasmid containing extraintestinal virulence genes is particularly worrisome. Massive and inappropriate use of veterinary antibiotics, such as tetracycline, in food-animal production promotes antimicrobial drug resistance among animals, known to be reservoirs for STEC. This practice can select and favor the spread of such MDR plasmids in human EHECs. Tetracycline still represented $36.5 \%$ of the tonnage of veterinary antibiotic use in 2015 in France (30). In our panel, all the sequenced O80 STEC strains carry the tetA gene, conferring resistance to tetracycline. Thus, large veterinary use of this drug might favor the selection of these hybrid strains and increase their diffusion.

We indicated a potential reservoir of these hybrid pathotype strains when we identified $3 \mathrm{O} 80: \mathrm{H} 2$ strains isolated from cattle that carry the same VFs and resistance genes as human strains. However, the presence of the CC165 strains in chickens suggest that this clonal complex is also adapted to poultry. An initial description of pS88-like plasmids in avian pathogenic $E$. coli strains reinforces this hypothesis (31). Moreover, the environmental survival of this clone in these potential reservoirs might be enhanced because of the resistance to mercury shared by all but 1 strain (36047), all carriers of the pS88-like plasmid, irrespective of their origin. Such resistance to heavy metals has been rarely described in EHEC strains (22).

We also detected an O80:H19-CC165 strain devoid of virulence genes, which might represent the ancestral precursor of $\mathrm{CC} 165$, and from which these hybrid pathotype strains might have been derived. This strain could be used for tracing the genetic history of this clone in future studies.

Our genetic description of the emerging hybrid pathotype E. coli $\mathrm{O} 80: \mathrm{H} 2$, associated with $\mathrm{O} 80$-related strains, reveals the outstanding capacity of $\mathrm{O} 80-\mathrm{CC} 165$ to acquire the combination of virulence genes involved in intestinal and extraintestinal pathogenicity and genes conferring broad antibiotic resistance, including extended-spectrum $\beta$-lactamase-encoding genes and those most recently identified, such as $m c r-1$. O80-CC165 strains, which are able to integrate multiple VFs with various consequences, MDR genes that encompass nearly all classes, and bacteriocins, represent a serious threat because of their exceptional versatility and should therefore be closely monitored in all countries in Europe. 


\section{Acknowledgments}

We thank Roger Stephan and Lothar Beutin for providing the strains from Switzerland and Germany, respectively.

This work was financed by Fonds d'Etudes et de Recherche du Corps Médical, AP-HP. Work in the Laboratorio de Referencia de Escherichia coli was financed by grant no. ED431C-2017-57 from Consellería de Cultura, Educación e Ordenación Universitaria (Xunta de Galicia) and the European Regional Development Fund. The funders had no role in study design, data collection and interpretation, or the decision to submit the work for publication.

\section{About the Author}

Dr. Cointe is a microbiologist working in the Parisian Public Hospital in France. Her research domain is pediatric infections, particularly the pathogenicity of intestinal Escherichia coli infections and the potential interplay between intraintestinal and extraintestinal E. coli.

\section{References}

1. Garg AX, Suri RS, Barrowman N, Rehman F, Matsell D, Rosas-Arellano MP, et al. Long-term renal prognosis of diarrheaassociated hemolytic uremic syndrome: a systematic review, meta-analysis, and meta-regression. JAMA. 2003;290:1360-70. http://dx.doi.org/10.1001/jama.290.10.1360

2. Schmidt H, Karch H, Beutin L. The large-sized plasmids of enterohemorrhagic Escherichia coli $\mathrm{O} 157$ strains encode hemolysins which are presumably members of the E. coli alpha-hemolysin family. FEMS Microbiol Lett. 1994;117:189-96.

3. Brunder W, Schmidt H, Karch H. EspP, a novel extracellular serine protease of enterohaemorrhagic Escherichia coli O157:H7 cleaves human coagulation factor V. Mol Microbiol. 1997;24:767-78. http://dx.doi.org/10.1046/j.1365-2958.1997.3871751.x

4. Bruyand M. Surveillance du syndrome hémolytique et urémique post-diarrhéique chez les enfants de moins de 15 ans en France en 2016 [cited $2016 \mathrm{Feb}$ 16]. http://invs.santepubliquefrance.fr/ content/download/138859/500759/version/1/file/Bilan SHU_2016.pdf

5. Blanco M, Blanco JE, Mora A, Dahbi G, Alonso MP, González EA, et al. Serotypes, virulence genes, and intimin types of Shiga toxin (verotoxin)-producing Escherichia coli isolates from cattle in Spain and identification of a new intimin variant gene $(e a e-\xi)$. J Clin Microbiol. 2004;42:645-51. http://dx.doi.org/10.1128/ JCM.42.2.645-651.2004

6. Wijnsma KL, Schijvens AM, Rossen JWA, Kooistra-Smid AMDM, Schreuder MF, van de Kar NCAJ. Unusual severe case of hemolytic uremic syndrome due to Shiga toxin 2d-producing E. coli O80:H2. Pediatr Nephrol. 2017;32:1263-8. http://dx.doi.org/ 10.1007/s00467-017-3642-3

7. Fierz L, Cernela N, Hauser E, Nüesch-Inderbinen M, Stephan R. Characteristics of Shigatoxin-producing Escherichia coli strains isolated during 2010-2014 from human infections in Switzerland. Front Microbiol. 2017;8:1471. http://dx.doi.org/10.3389/ fmicb.2017.01471

8. Bielaszewska M, Mellmann A, Zhang W, Köck R, Fruth A, Bauwens A, et al. Characterisation of the Escherichia coli strain associated with an outbreak of haemolytic uraemic syndrome in Germany, 2011: a microbiological study. Lancet Infect Dis. 2011;11:671-6. http://dx.doi.org/10.1016/S1473-3099(11)70165-7
9. Mariani-Kurkdjian P, Lemaître C, Bidet P, Perez D, Boggini L, Kwon T, et al. Haemolytic-uraemic syndrome with bacteraemia caused by a new hybrid Escherichia coli pathotype. New Microbes New Infect. 2014;2:127-31. http://dx.doi.org/10.1002/nmi2.49

10. Soysal N, Mariani-Kurkdjian P, Smail Y, Liguori S, Gouali M, Loukiadis E, et al. Enterohemorrhagic Escherichia coli hybrid pathotype O80:H2 as a new therapeutic challenge. Emerg Infect Dis. 2016;22:1604-12. http://dx.doi.org/10.3201/eid2209.160304

11. Peigne C, Bidet P, Mahjoub-Messai F, Plainvert C, Barbe V, Médigue C, et al. The plasmid of Escherichia coli strain S88 $(\mathrm{O} 45: \mathrm{K} 1: \mathrm{H} 7)$ that causes neonatal meningitis is closely related to avian pathogenic $E$. coli plasmids and is associated with high-level bacteremia in a neonatal rat meningitis model. Infect Immun. 2009;77:2272-84. http://dx.doi.org/10.1128/IAI.01333-08

12. Carattoli A, Zankari E, García-Fernández A, Voldby Larsen M, Lund $\mathrm{O}$, Villa L, et al. In silico detection and typing of plasmids using PlasmidFinder and plasmid multilocus sequence typing. Antimicrob Agents Chemother. 2014;58:3895-903.

http://dx.doi.org/10.1128/AAC.02412-14

13. Kaas RS, Leekitcharoenphon P, Aarestrup FM, Lund O. Solving the problem of comparing whole bacterial genomes across different sequencing platforms. PLoS One. 2014;9:e104984. http://dx.doi.org/10.1371/journal.pone.0104984

14. Joensen KG, Tetzschner AMM, Iguchi A, Aarestrup FM, Scheutz F. Rapid and easy in silico serotyping of Escherichia coli isolates by use of whole-genome sequencing data. J Clin Microbiol. 2015;53:2410-26. http://dx.doi.org/10.1128/JCM.00008-15

15. Wirth T, Falush D, Lan R, Colles F, Mensa P, Wieler LH, et al. Sex and virulence in Escherichia coli: an evolutionary perspective. Mol Microbiol. 2006;60:1136-51. http://dx.doi.org/10.1111/ j.1365-2958.2006.05172.x

16. Zankari E, Hasman $H$, Cosentino S, Vestergaard M, Rasmussen S, Lund $\mathrm{O}$, et al. Identification of acquired antimicrobial resistance genes. J Antimicrob Chemother. 2012;67:2640-4. http://dx.doi.org/ 10.1093/jac/dks261

17. Bielaszewska M, Friedrich AW, Aldick T, Schürk-Bulgrin R, Karch H. Shiga toxin activatable by intestinal mucus in Escherichia coli isolated from humans: predictor for a severe clinical outcome. Clin Infect Dis. 2006;43:1160-7. http://dx.doi.org/10.1086/508195

18. Serra-Moreno R, Jofre J, Muniesa M. Insertion site occupancy by $s t x_{2}$ bacteriophages depends on the locus availability of the host strain chromosome. J Bacteriol. 2007;189:6645-54. http://dx.doi.org/10.1128/JB.00466-07

19. Bonanno L, Loukiadis E, Mariani-Kurkdjian P, Oswald E, Garnier L, Michel V, et al. Diversity of Shiga toxin-producing Escherichia coli (STEC) O26:H11 strains examined via stx subtypes and insertion sites of Stx and EspK bacteriophages. Appl Environ Microbiol. 2015;81:3712-21. http://dx.doi.org/10.1128/AEM.00077-15

20. Recktenwald J, Schmidt H. The nucleotide sequence of Shiga toxin (Stx) 2e-encoding phage phiP27 is not related to other Stx phage genomes, but the modular genetic structure is conserved. Infect Immun. 2002;70:1896-908. http://dx.doi.org/10.1128/ IAI.70.4.1896-1908.2002

21. Falgenhauer L, Yao Y, Fritzenwanker M, Schmiedel J, Imirzalioglu C, Chakraborty T. Complete genome sequence of phage-like plasmid pECOH89, encoding CTX-M-15. Genome Announc. 2014; 2:e00356-14. http://dx.doi.org/10.1128/genomeA.00356-14

22. Venturini C, Hassan KA, Roy Chowdhury P, Paulsen IT, Walker MJ, Djordjevic SP. Sequences of two related multiple antibiotic resistance virulence plasmids sharing a unique IS26-related molecular signature isolated from different Escherichia coli pathotypes from different hosts. PLoS One. 2013;8:e78862. http://dx.doi.org/10.1371/journal.pone.0078862

23. Feng P, Lampel KA, Karch H, Whittam TS. Genotypic and phenotypic changes in the emergence of Escherichia coli O157:H7. J Infect Dis. 1998;177:1750-3. http://dx.doi.org/10.1086/517438 
24. Jost C, Bidet P, Carrère T, Mariani-Kurkdjian P, Bonacorsi S. Susceptibility of enterohaemorrhagic Escherichia coli to azithromycin in France and analysis of resistance mechanisms. J Antimicrob Chemother. 2016;71:1183-7. http://dx.doi.org/ 10.1093/jac/dkv477

25. Liu Y-Y, Wang Y, Walsh TR, Yi L-X, Zhang R, Spencer J, et al. Emergence of plasmid-mediated colistin resistance mechanism MCR-1 in animals and human beings in China: a microbiological and molecular biological study. Lancet Infect Dis. 2016;16:161-8. http://dx.doi.org/10.1016/S1473-3099(15)00424-7

26. Donà V, Bernasconi OJ, Pires J, Collaud A, Overesch G, Ramette A, et al. Heterogeneous genetic location of $\mathrm{mcr}^{-1}$ in colistin-resistant Escherichia coli isolates from humans and retail chicken meat in Switzerland: emergence of $m c r-1$-carrying IncK2 plasmids. Antimicrob Agents Chemother. 2017;61:e01245-17. http://dx.doi.org/10.1128/AAC.01245-17

27. Day M, Doumith M, Jenkins C, Dallman TJ, Hopkins KL, Elson R, et al. Antimicrobial resistance in Shiga toxin-producing Escherichia coli serogroups $\mathrm{O} 157$ and $\mathrm{O} 26$ isolated from human cases of diarrhoeal disease in England, 2015. J Antimicrob Chemother. 2017;72:145-52. http://dx.doi.org/10.1093/jac/dkw371

28. Freedman SB, Xie J, Neufeld MS, Hamilton WL, Hartling L, Tarr PI; Alberta Provincial Pediatric Enteric Infection Team (APPETITE). Shiga toxin-producing Escherichia coli infection, antibiotics, and risk of developing hemolytic uremic syndrome: a meta-analysis. Clin Infect Dis. 2016;62:1251-8. http://dx.doi.org/10.1093/cid/ciw099

29. Fricke WF, McDermott PF, Mammel MK, Zhao S, Johnson TJ, Rasko DA, et al. Antimicrobial resistance-conferring plasmids with similarity to virulence plasmids from avian pathogenic Escherichia coli strains in Salmonella enterica serovar Kentucky isolates from poultry. Appl Environ Microbiol. 2009;75:5963-71. http://dx.doi.org/10.1128/AEM.00786-09

30. Agence Nationale de Sécurité Sanitaire de l'Alimentation, de l'Environnement et du Travail (ANSES). Suivi des ventes de médicaments vétérinaires contenant des antibiotiques en France en 2015 [cited 2016 Feb 16]. https://www.anses.fr/fr/system/files/ ANMV-Ra-Antibiotiques2015.pdf

31. Johnson TJ, Siek KE, Johnson SJ, Nolan LK. DNA sequence of a ColV plasmid and prevalence of selected plasmid-encoded virulence genes among avian Escherichia coli strains. J Bacteriol. 2006;188:745-58. http://dx.doi.org/10.1128/ JB.188.2.745-758.2006

Address for correspondence: Stéphane Bonacorsi, Service de Microbiologie, CHU Robert Debré, 48 boulevard Sérurier, 75019 Paris, France; email: stephane.bonacorsi@aphp.fr

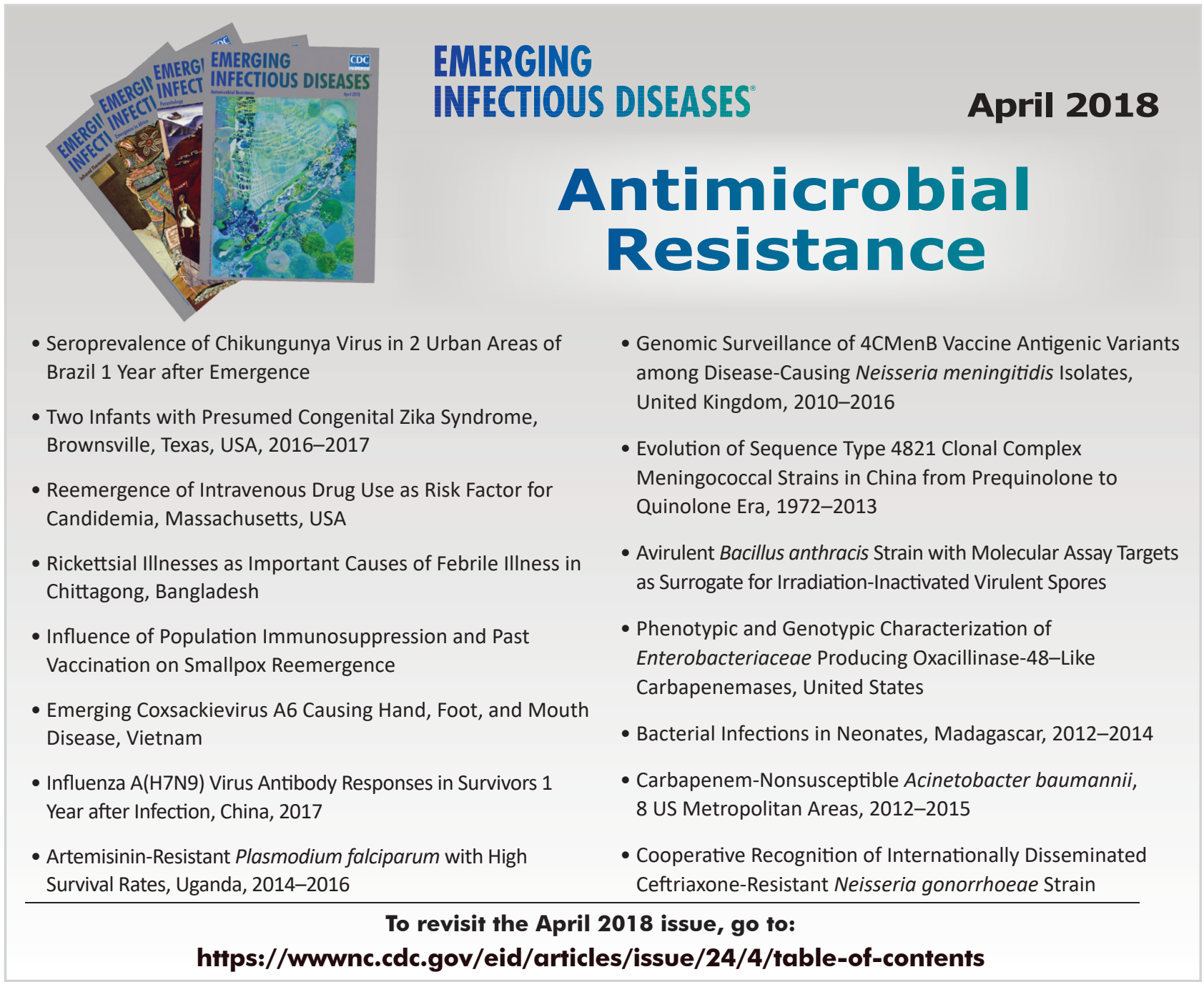

